\title{
Molecular epidemiological tracing of a cattle rabies outbreak lasting less than a month in Rio Grande do Sul in southern Brazil
}

\author{
Takuya Itou ${ }^{1 *}$, Toshiharu Fukayama ${ }^{1}$, Nobuyuki Mochizuki ${ }^{1}$, Yuki Kobayashi $^{1}$, Eduardo R. Deberaldini ${ }^{2}$, \\ Adolorata A. B. Carvalho², Fumio H. Ito ${ }^{3}$ and Takeo Sakai ${ }^{1}$
}

\begin{abstract}
Background: Vampire bat-transmitted cattle rabies cases are typically encountered in areas where the disease is endemic. However, over the period of a month in 2009, an outbreak of cattle rabies occurred and then ended spontaneously in a small area of the Rio Grande do Sul State in southern Brazil. To investigate the epidemiological characteristics of this rabies outbreak in Rio Grande do Sul, 26 nucleotide sequences of rabies virus (RABV) genomes that were collected in this area were analyzed phylogenetically.
\end{abstract}

Results: Nucleotide sequence identities of the nucleoprotein gene and $G-L$ intergenic region of the 26 RABVs were greater than $99.6 \%$. Phylogenetic analysis showed that all RABVs clustered with the vampire bat-related cattle RABV strains and that the RABVs were mainly distributed in southern Brazil.

Conclusions: The findings of the present study suggested that a small population of rabid vampire bats carrying a single RABV strain produced a spatiotemporally restricted outbreak of cattle rabies in southern Brazil.

Keywords: Brazil, Cattle rabies, Molecular epidemiology, Vampire bat

\section{Background}

Rabies is a fatal infectious disease that causes encephalomyelitis. Genetic diversity among rabies viruses (RABVs) depends on the host species and its geographic distribution. Rabies is endemic in Brazil, and RABVs have been isolated from a variety of mammalian hosts, including dogs, foxes, cats, cattle, and hematophagous, insectivorous and frugivorous bats [1-3]. Vampire bats, particularly Desmodus rotundus, are important vectors of rabies in Latin America. Rabies transmission from vampire bats to humans occurs frequently, primarily in the Amazonian regions of Brazil and Peru, and cattle rabies transmitted by vampire bats is also common in Brazil [4]. Vampire bat-related rabies is thus a serious problem from both animal and public health standpoints.

\footnotetext{
*Correspondence: itou.takuya@nihon-u.ac.jp

${ }^{1}$ Nihon University Veterinary Research Center, Nihon University, 1866

Kameino, Fujisawa, Kanagawa 252-0880, Japan

Full list of author information is available at the end of the article
}

Molecular and geographic epidemiological analyses of livestock rabies in northeastern, central and southeastern Brazil revealed that RABVs isolated from livestock were closely related to the virus strains found in vampire bat populations, and that the RABVs in these areas were stable within a spatiotemporal context [5]. However, relatively little information currently exists on the molecular epidemiology of RABVs isolated from livestock in southern Brazil.

The incidence of vampire bat-transmitted cattle rabies cases is typically restricted to areas where the disease is endemic [5-7]. However, between February and March 2009, an outbreak of cattle rabies occurred and ended spontaneously in a small region of Rio Grande do Sul State (RS) in southern Brazil. To investigate the epidemiological characteristics of this rabies outbreak in RS, the RABVs obtained in this area were subjected to phylogenetic analysis. 


\section{Methods}

An outbreak of cattle rabies occurred in a hilly area $(<5 \mathrm{~km}$ radius) in the city of Nova Roma do Sul in Rio Grande do Sul State, southern Brazil (Fig. 1). This outbreak occurred on a total of 19 cattle farms between February and March 2009, and ended spontaneously without any mitigation measures being implemented. Outbreaks of cattle rabies in this region had not been observed for at least 3 years before or after this outbreak. Brain specimens from these livestock were diagnosed as RABV-positive by an immunofluorescent antibody test and viral RNA was extracted from the brains of livestock using the QIAamp Viral RNA Mini Kit (Qiagen, Hilden, Germany). Nucleoprotein (N) gene sequences from the Brazilian RABV samples were amplified by RT-PCR and determined using applied biosystems 3130 genetic analyzer (ABI) using primers described previously $[8,9]$. Information of primers used for RT-PCR and sequencing are shown in Additional file 1: Table S1. Multiple alignment for the datasets of $\mathrm{N}$ and $\mathrm{G}-\mathrm{L}$ genes were made by MAFFT program [10]. Neighbor-joining tree of $\mathrm{N}$ gene with 1000 bootstrap replicates was constructed with the p-distance using MEGA ver 6.10 [11]. The nucleotide sequences retrieved from GenBank to construct the phylogenetic tree are listed in Additional file 2: Table S2.

The geographic origins of the RABV sequences obtained from the Brazilian livestock were plotted at the municipal level in the federal states in which infection was observed using MapInfo Professional GIS software (ver. 8.0, MapInfo Japan K.K., Tokyo, Japan) and Google Earth (ver.6.0, Google, Mountain View, CA, USA). Brazilian maps were obtained from Brasil em RelevoEmbrapa Monitoramento por Satélite [12].

\section{Results}

A total of 26 RNA samples were extracted from brains of rabid cattle from the 19 farms surveyed in this study (Fig. 1; Table 1). The PCR amplified sequences of the 1424-nt N-mRNA region and 578-nt G-L region were determined for all 26 RABV samples [GenBank: AB685222-AB685247 and LC057591-LC057616] (Table 1). The results from $\mathrm{N}$ gene sequences showed that, with the exception of BRbv1289, the nucleotide and amino acid sequence identities of the RS RABVs were $100 \%$. Between BRbv1285, which was selected among the 25 identical RABV RNAs as the representative sequence, and BRbv1289, nucleotide and amino acid sequence identities were more than 99.9 and $100 \%$, respectively. To validate the genomic homogeneity among the RABV samples, the variable $\mathrm{G}-\mathrm{L}$ intergenic 578-bp region (position: 4929-5514 of PV) was additionally analyzed. The nucleotide sequence identity was more than $99.6 \%$ among all 26 RABVs, showing the quite high homogeneity of epidemic RABVs in this outbreak.

Phylogenetic analysis based on a partial $\mathrm{N}$ sequence (position: 203-1420 of PV) revealed that 26 RABV samples formed a monophyletic cluster in vampire batrelated RABV lineage (Fig. 2). Comparison with RABVs obtained from neighboring countries, indicated that these 26 RABVs formed a sub-cluster with RABVs collected in the states of Sao Paulo (SP) and Rio de Janeiro (RJ) in Brazil. In addition to this sub-cluster, there are two another sub-clusters consisted of RABV samples collected in the state of SP and neighboring areas. These lineages were named the lineages of RD1, RD2/3, and Old strains by Carnieli et al. [13]. RS strains belonged to the lineage of Old strains and shared a common ancestor with RD1 lineage. RABVs in RD1 and RD2/3 lineages were distributed in mountain range located in the boundary of the states of SP, Minas Gerais, and RJ, while Old strains were isolated from towns in the interior of the state of SP [13].

Using a 203-bp segment (position: 109-311 of PV) of the $\mathrm{N}$ gene, Kobayashi et al. reported the existence of at least 24 RABV genetic variants among the vampire battransmitted rabies cases in cattle in Brazil [5]. Using the same 203-bp segment, our analysis revealed that the 26 RS RABVs of the present study correlate with the C-19 lineage described by Kobayashi et al. [5] (Additional file 3: Figure S1). Samples belonging to the C-19 lineage were distributed in the states of RS, Goiás, Mato Grosso do Sul, SP and RJ, which are the southeastern regions of Brazil [5].

\section{Discussion}

Nucleoprotein gene and G-L intergenic segments from the 26 RABV samples collected in early spring of 2009 from separate farms in a restricted region of Nova Roma do Sul, RS, were sequenced and subjected to phylogenetic analysis. The genetic analysis revealed that the nucleotide and amino acid sequences were almost identical among the $26 \mathrm{RABVs}$ and shared a common ancestor, suggesting the RABV strain was transmitted from a small population of rabid vampire bats infected with RABV, which may be derived from a single introduction, to a cattle population grazing in Nova Roma do Sul.

Generally, vampire bat-transmitted RABVs were restricted to specific geographic areas because of the vampire bat ecology. The phylogenetic analysis described here revealed that all of the livestock RABVs in RS were derived from vampire bat rabies. Although we were unable to investigate the prevalence of rabid vampire bats in this area, the findings of the present study revealed that the 26 RABVs correspond with the "Old strains" lineage 


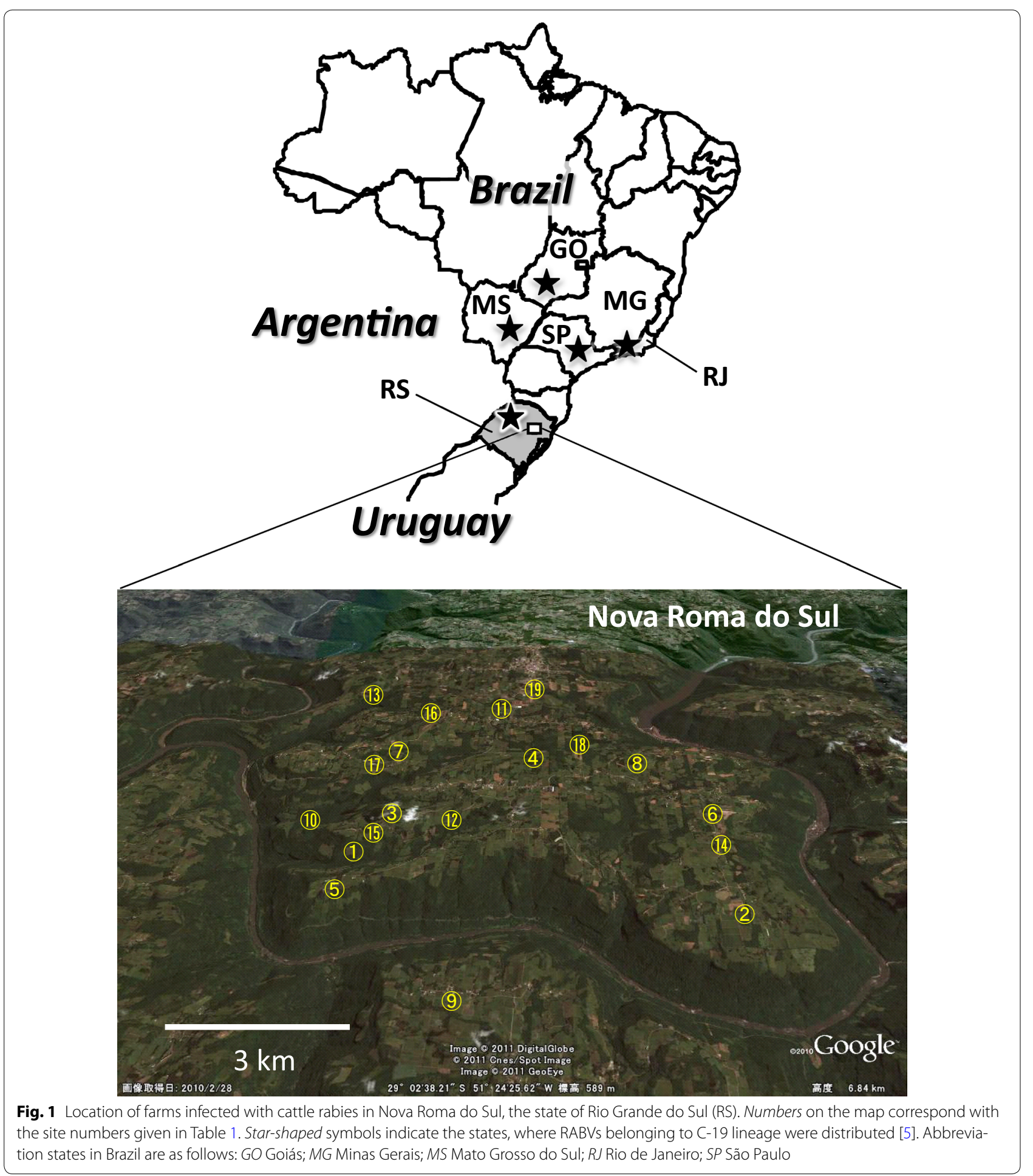

reported by Carnieli Jr et al. and C-19 lineage identified by Kobayashi et al. $[5,13]$. The distribution of RABV lineages in Brazil is known to be delimited by geomorphological boundaries, such as mountain ranges and rivers [14]. The reason why the $\mathrm{C}-19$ lineage consists of RABVs from
Brazil and none from Argentina may be thus because a very large river, the Uruguay River, separates the Brazil and Argentina. However, the C-19 lineage is distributed over larger areas. Since the "Old strains" lineage, which was made up basically of the isolates collected before 
Table 1 Rabies virus samples obtained from the Rio Grande do Sul

\begin{tabular}{|c|c|c|c|c|}
\hline \multirow{2}{*}{$\begin{array}{l}\text { Sample } \\
\text { name }\end{array}$} & \multirow{2}{*}{$\begin{array}{l}\text { Date } \\
\text { of sampling }\end{array}$} & \multirow{2}{*}{$\begin{array}{l}\text { Site } \\
\text { of occur- } \\
\text { rence }^{b}\end{array}$} & \multicolumn{2}{|c|}{ GenBank accession \# } \\
\hline & & & N gene & G-L intergenic \\
\hline BRbv1285 & 28/02/2009 & 1 & AB685222 & LC057591 \\
\hline BRbv1286 & 08/03/2009 & 2 & AB685223 & LC057592 \\
\hline BRbv1287 & 18/03/2009 & 3 & AB685224 & LC057593 \\
\hline BRbv1288 & 28/02 2009 & 4 & AB685225 & LC057594 \\
\hline BRbv1289 & 28/02/2009 & 5 & AB685226 & LC057595 \\
\hline BRbv1290 & 28/02/2009 & 6 & AB685227 & LC057596 \\
\hline BRbv1291 & 23/02/2009 & 1 & AB685228 & LC057597 \\
\hline BRbv1292 & $11 / 03 / 2009$ & 7 & AB685229 & LC057598 \\
\hline BRbv1293 & 13/03/2009 & 8 & AB685230 & LC057599 \\
\hline BRbv1294 & 06/03/2009 & 6 & AB685231 & LC057600 \\
\hline BRbv1297 & 08/03/2009 & 9 & AB685232 & LC057601 \\
\hline BRbv1298 & 09/03/2009 & 10 & AB685233 & LC057602 \\
\hline BRbv1299 & 12/03/2009 & 1 & AB685234 & LC057603 \\
\hline BRbv1301 & 18/02/2009 & 11 & AB685235 & LC057604 \\
\hline BRbv1302 & 13/03/2009 & 12 & AB685236 & LC057605 \\
\hline BRbv1303 & 18/03/2009 & 3 & AB685237 & LC057606 \\
\hline BRbv1304 & 18/03/2009 & 13 & AB685238 & LC057607 \\
\hline BRbv1305 & 23/02/2009 & 2 & AB685239 & LC057608 \\
\hline BRbv1306 & $--^{a}$ & 14 & AB685240 & LC057609 \\
\hline BRbv1307 & 12/03/2009 & 15 & AB685241 & LC057610 \\
\hline BRbv1308 & 27/02/2009 & 16 & AB685242 & LC057611 \\
\hline BRbv1309 & 02/03/2009 & 17 & AB685243 & LC057612 \\
\hline BRbv1310 & 23/02/2009 & 18 & AB685244 & LC057613 \\
\hline BRbv1311 & 25/02/2009 & 19 & AB685245 & LC057614 \\
\hline BRbv1312 & 28/02/2009 & 5 & AB685246 & LC057615 \\
\hline BRbv1313 & 04/03/2009 & 9 & AB685247 & LC057616 \\
\hline
\end{tabular}

a No information

b Numbers correspond to those in Fig. 1

1998, has been endemic in the state of SP before epizootic of RD2/RD3 were observed [13], suggesting that RABVs in Old strains lineages may have gradually spread in southern Brazil, including the state of RS.

The typical distribution range of $D$. rotundas activity around a roost is about $10 \mathrm{~km}^{2}$ [15]; the range of this outbreak was approximately $80 \mathrm{~km}^{2}$. In extensive random surveys conducted in Trinidad and Brazil, the prevalence of the virus among vampire bats in the field was 0.46$0.75 \%$ (see Baer) [16]. Further, no rabies outbreaks have been reported in the past 3 years in this region, and the outbreak ended without any specific prevention measures being implemented for cattle rabies. Although the non-migratory, vampire bats have distribution ranges of between 10 and $20 \mathrm{~km}^{2}$ [17], it has been suggested that the vampire bats may need to fly over longer distances to feed and that they share roosts with other bat species;

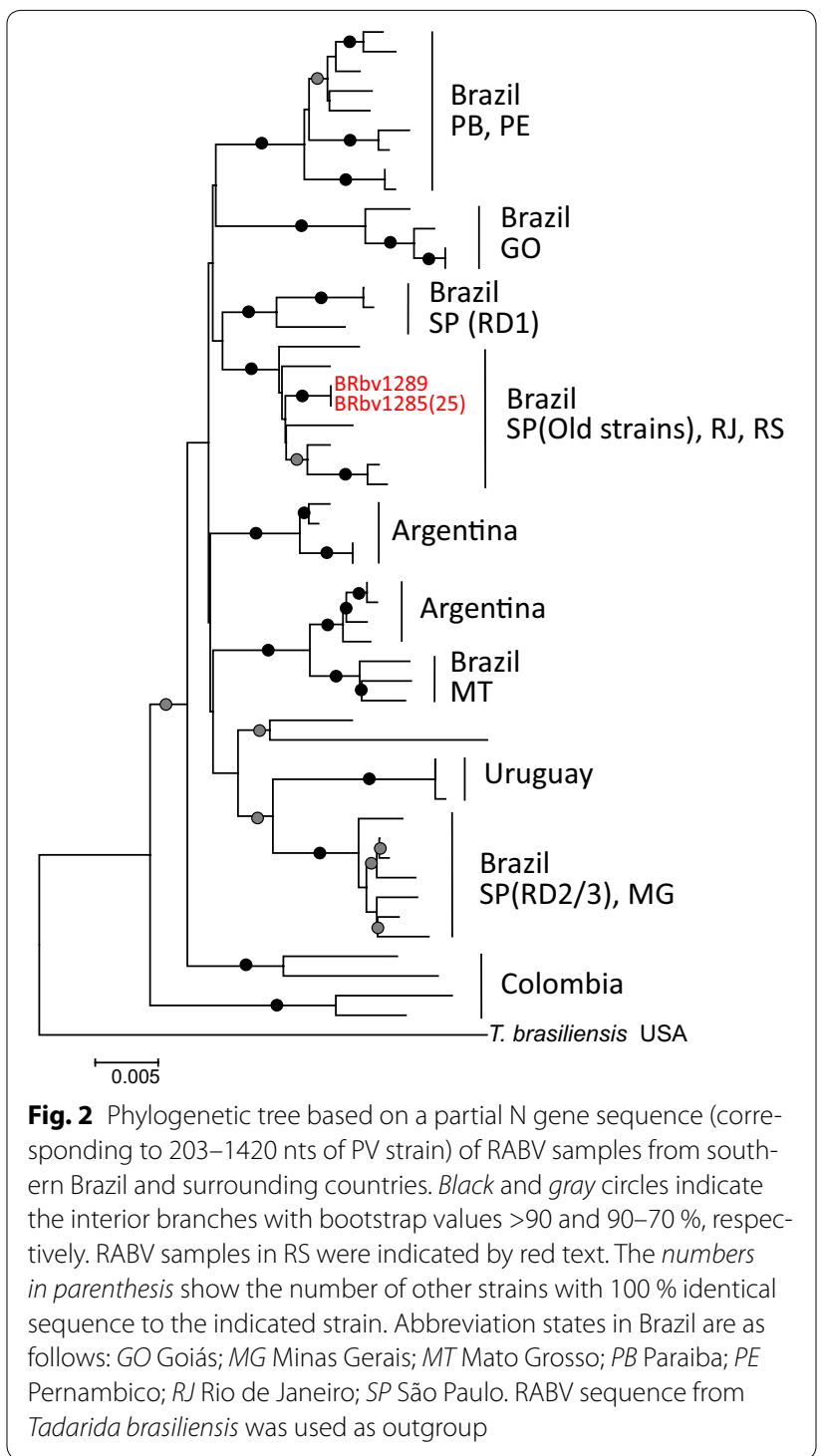

indeed, this may explain how the RABV could spread over a short period of time [18]. However, the cattle rabies cases described in the present study ended suddenly over the period of a month. There are several possible reasons why the activities of the vampire bats may have been limited and why this outbreak of cattle rabies ended suddenly. The affected area was geographically isolated by a river valley that may have limited the feeding activities and distribution of the bats. It is also possible that the size of the vampire bat population in the region may have been too small to act as reservoir for RABV.

Taken together, it is considered that this outbreak was caused by small population of vampire bats that were vectors of the RABV lineage that is distributed mainly in southern Brazil. It is considered that the relatively 
restricted range of bats contributed to the sudden end of the outbreak before it could spread to other regions.

\section{Conclusions}

Segments of $\mathrm{N}$ gene and $\mathrm{G}-\mathrm{L}$ intergenic region sequences from $26 \mathrm{RABV}$ samples that were collected in early spring from separate farms in small region of Nova Roma do Sul, Rio Grande do Sul, in 2009, were subjected to phylogenetic analysis. The $\mathrm{N}$ gene and $\mathrm{G}-\mathrm{L}$ region sequence analysis revealed that the $26 \mathrm{RABVs}$ had almost identical nucleotide and amino acid sequences. The outbreak was likely caused by a small population of vampire bats that were infected with a single strain of the RABV lineage found mainly in southern Brazil that invaded the area of the study site.

\section{Additional files}

Additional file 1. Primers used for RT-PCR and sequencing.

Additional file 2. Nucleotide sequences RABV N gene retrieved from GenBank.

Additional file 3. Phylogenetic tree based on nucleoprotein gene 203 nts (corresponding 107-311 nts of PV strain) using Brazilian RABV isolates. The 24 lineages classified by Kobayashi et al. [5] and RS RABV samples in this study were compared. All RS samples belonged to C-19 lineage maintained in Southwestern Brazil. The symbols for each lineage correspond to the results by Kobayashi et al. [5]

\section{Abbreviation}

RABV: rabies virus.

\section{Authors' contributions}

ERD and AASBC collected epidemiological samples and information in the field. TI, TF and NM drafted the manuscript. TF conducted the RT-PCR and sequencing analyses. TI, NM, YK, FHI and TS contributed to study design, management, coordination, and drafting of the manuscript. All authors read and approved the final manuscript.

\section{Author details}

${ }^{1}$ Nihon University Veterinary Research Center, Nihon University, 1866 Kameino, Fujisawa, Kanagawa 252-0880, Japan. ${ }^{2}$ Department of Preventive Veterinary Medicine, Faculty of Agriculture and Veterinary Science, UNESP, Via de Acesso Prof. Paulo Donato Castellane, Jaboticabal, Sao Paulo 14884-900, Brazil. ${ }^{3}$ Department of Preventive Veterinary Medicine and Animal Health, Faculty of Veterinary Medicine and Zootechny, University of São Paulo, Av. Prof. Dr. Orlando Marques de paiva, 87, Cidade Universtiátria, Sao Paulo, São Paulo 05508-000, Brazil.

\section{Acknowledgements}

This study was supported in part by a Grant-in-Aid for Scientific Research (24580453) from the Japan Society for the Promotion of Science and the Strategic Research Base Development Program, "International joint research and training of young researchers for zoonosis control in a globalized world", and a matching fund subsidy from the Ministry of Education, Culture, Sports, Science and Technology of Japan (S0991023 and S1491007).

\section{Competing interests}

The authors declare that they have no competing interests.

Received: 5 August 2015 Accepted: 2 February 2016

Published online: 12 February 2016

\section{References}

1. Ito M, Arai YT, Itou T, Sakai T, Ito FH, Takasaki T, Kurane I. Genetic characterization and geographic distribution of rabies virus isolates in Brazil: identification of two reservoirs, dogs and vampire bats. Virology. 2001;284:214-22.

2. Sato G, Kobayashi Y, Shoji Y, Sato T, Itou T, Ito FH, Santos HP, Brito CJ, Sakai T. Molecular epidemiology of rabies from Maranhão and surrounding states in the northeastern region of Brazil. Arch Virol. 2006;151:2243-51.

3. Kobayashi Y, Sato G, Kato M, Itou T, Cunha EM, Silva MV, Mota CS, Ito $\mathrm{FH}$, Sakai T. Genetic diversity of bat rabies viruses in Brazil. Arch Virol. 2007;152:1995-2004.

4. Kobayashi Y, Ogawa A, Sato G, Sato T, Itou T, Samara SI, Carvalho AA, Nociti DP, Ito FH, Sakai T. Geographical distribution of vampire bat-related cattle rabies in Brazil. J Vet Med Sci. 2006:68:1097-100.

5. Kobayashi Y, Sato G, Mochizuki N, Hirano S, Itou T, Carvalho AA, Albas A, Santos HP, Ito FH, Sakai T. Molecular and geographic analyses of vampire bat-transmitted cattle rabies in central Brazil. BMC Vet Res. 2008;4:44.

6. Condori-Condori RE, Streicker DG, Cabezas-Sanchez C, Velasco-Villa A. Enzootic and epizootic rabies associated with vampire bats, peru. Emerg Infect Dis. 2013;19(9):1463-9.

7. Torres C, Lema C, Dohmen FG, Beltran F, Novaro L, Russo S, Freire MC, Velasco-Villa A, Mbayed VA, Cisterna DM. Phylodynamics of vampire battransmitted rabies in Argentina. Mol Ecol. 2014;23:2340-52.

8. Hirano S, Itou T, Carvalho AA, Ito FH, Sakai T. Epidemiology of vampire bat-transmitted rabies virus in Goiás, central Brazil: re-evaluation based on $\mathrm{G}-\mathrm{L}$ intergenic region. BMC Res Notes. 2012;3:288.

9. Mochizuki N, Kobayashi Y, Sato G, Hirano S, Itou T, Ito FH, Sakai T. Determination and molecular analysis of the complete genome sequence of two wild-type rabies viruses isolated from a haematophagous bat and a frugivorous bat in Brazil. J Vet Med Sci. 2011;73:759-66.

10. Katoh K, Standley DM. MAFFT multiple sequence alignment software version 7: improvements in performance and usability. Mol Biol Evol. 2013;30:772-80

11. Tamura K, Stecher G, Peterson D, Filipski A, Kumar S. MEGA6: molecular evolutionary genetics analysis version 6.0. Mol Biol Evol. 2013;30:2725-9.

12. Brasil em Relevo_Embrapa Monitoramento por Satélite http://www. relevobr.cnpm.embrapa.br/. Accessed 13 May 2015.

13. Carnieli P Jr, Castilho JG, Fahl Wde O, Véras NM, Timenetsky Mdo C. Genetic characterization of rabies virus isolated from cattle between 1997 and 2002 in an epizootic area in the state of São Paulo, Brazil. Virus Res. 2009;144:215-24.

14. Mochizuki N, Kawasaki H, Silva ML, Afonso JA, Itou T, Ito FH, Sakai T. Molecular epidemiology of livestock rabies viruses isolated in the northeastern Brazilian states of Paraíba and Pernambuco from 2003-2009. BMC Res Notes. 2012;5:32.

15. Arellano-Sota C. Biology, ecology, and control of the vampire bat. Rev Infect Dis. 1988;10(Suppl 4):S615-9.

16. Baer GM. Vampire bat and bovine paralytic rabies. In: Baer GM, editor. Natural History of Rabies. 2nd ed. Boston: CRC Press; 1991. p. 389-403.

17. Lord RD. Seasonal reproduction of vampire bats and its relation to seasonality of bovine rabies. J Wildl Dis. 1992;28:292-4.

18. Romijn PC, van der Heide R, Cattaneo CA, Silva Rde C, van der Poel WH. Study of lyssaviruses of bat origin as a source of rabies for other animal species in the State of Rio De Janeiro, Brazil. Am J Trop Med Hyg. 2003;69:81-6. 